\title{
Assistência de enfermagem em pacientes com síndrome de HELLP
}

\author{
Nursing care in patients with HELLP syndrome \\ Cuidados de enfermería en pacientes con síndrome HELLP
}

\section{Resumo}

A síndrome HELLP é caracterizada por meio de diagnóstico primário de pré-eclâmpsia, caracterizada pela ocorrência da elevação da pressão arterial na gestação em torno da $20^{\mathrm{a}}$ semana, acompanhada de edema e proteinúria. A síndrome de HELLP é muito grave e aumenta o risco de morte para o feto e a gestante. O objetivo central deste estudo é descrever os cuidados de enfermagem na gestante com síndrome de HELLP e, mais especificamente, descrever as principais complicações da síndrome de HELLP na gestante. O estudo foi realizado por meio de uma revisão bibliográfica de abordagem descritiva e qualitativa. Os principais cuidados de enfermagem na gestante com HELLP identificados na literatura analisada foram: monitorizar a frequência respiratória, saturação de oxigênio, débito urinário e reflexos patelares, manter o repouso, pesar diariamente, realizar controle hidroeletrolítico e controlar os batimentos fetais. A síndrome de HELLP pode apresentar complicações cerebrovasculares, hemorragia, edema pulmonar, descolamento da retina, hematoma/hepática, insuficiência renal aguda, insuficiência hepática, insuficiência coagulopatia, desprendimento placentário, sepse, ativação plaquetária, elevação níveis séricos ocitocinas, obstrução sinusoidal e infarto hepático, hemorragia hepática, contrações uterinas, convulsões e hemólise.

Palavras-chave: Atenção à saúde; Cuidados de enfermagem; Síndrome HELLP.

\begin{abstract}
HELLP syndrome is characterized by primary diagnosis of pre-eclampsia, characterized by the occurrence of elevated blood pressure during pregnancy around the 20th week of pregnancy, accompanied by edema and proteinuria. HELLP syndrome is very serious and increases the risk of death for both the fetus and the pregnant woman. The main objective of this study is to describe nursing care for pregnant women with HELLP syndrome and, more specifically, to describe the main complications of HELLP syndrome in pregnant women. The study was carried out through a literature review with a descriptive and qualitative approach. The main nursing care measures for pregnant women with HELLP identified in the literature analyzed were monitoring respiratory rate, oxygen saturation, urinary output, and patellar reflexes, maintaining rest, weighing daily, performing hydroelectrolyte control and controlling fetal heart rate. HELLP syndrome can present cerebrovascular complications, hemorrhage, pulmonary edema, retinal detachment, hematoma/hepatic, acute renal failure, liver failure, coagulopathy failure, placental detachment, sepsis, platelet activation, elevated serum oxytocin levels, sinusoidal obstruction and hepatic infarction, hepatic hemorrhage, uterine contractions, convulsions, and hemolysis.
\end{abstract}

Keywords: Health care; Nursing care; HELLP Syndrome.

\section{Resumen}

El síndrome HELLP se caracteriza por el diagnóstico primario de preeclampsia, caracterizado por la aparición de hipertensión arterial durante el embarazo alrededor de la semana 20, acompañada de edema y proteinuria. El síndrome 
HELLP es muy grave y aumenta el riesgo de muerte tanto para el feto como para la mujer embarazada. El objetivo principal de este estudio es describir la atención de enfermería a la gestante con síndrome HELLP y, más específicamente, describir las principales complicaciones del síndrome HELLP en la gestante. El estudio se llevó a cabo mediante una revisión de la literatura con un enfoque descriptivo y cualitativo. Las principales medidas de atención de enfermería para gestantes con HELLP identificadas en la literatura analizada fueron: monitoreo de la frecuencia respiratoria, saturación de oxígeno, gasto urinario y reflejos rotulianos, mantenimiento del reposo, pesaje diario, realización de control hidroelectrolítico y control de la frecuencia cardíaca fetal. El síndrome HELLP puede presentar complicaciones cerebrovasculares, hemorragia, edema pulmonar, desprendimiento de retina, hematoma / hepático, insuficiencia renal aguda, insuficiencia hepática, insuficiencia de coagulopatía, desprendimiento de placenta, sepsis, activación plaquetaria, niveles elevados de oxitocina sérica, obstrucción sinusoidal e infarto hepático, hemorragia hepática, contracciones uterinas, convulsiones y hemólisis.

Palabras clave: Cuidado de la salud; Cuidado de enfermera; Síndrome de HELLP.

\section{Introdução}

No decorrer da gestação é necessário o acompanhamento pré-natal realizado em unidade de saúde, por meio de consultas com a equipe multiprofissional. Este momento é importante para prevenir e identificar precocemente os problemas que podem ocorrer durante o período gestacional. Um dos principais problemas que requer atenção é a síndrome hipertensiva específica da gestação (SHEG), que apresenta como consequência gravíssima a Síndrome de HELLP, do inglês hemolysis, elevated liver enzimes, and low platelet count, que significa hemólise, enzimas hepáticas elevadas, baixa contagem de plaquetas). Esta síndrome leva a alterações significativas nos exames clínicos com aumento da hemólise, disfunção hepática e plaquetopenia.

A síndrome HELLP é caracterizada por meio de diagnóstico primário de pré-eclâmpsia, caracterizada pela ocorrência da elevação da pressão arterial na gestação em torno da $20^{\mathrm{a}}$ semana, acompanhada de edema e proteinúria. A síndrome de HELLP é muito grave e aumenta o risco de morte para o feto e a gestante.

Normalmente para realizar a prevenção de complicações e acompanhamento adequado da SHEG, a gestante deve ser atendida nas unidades de saúde por meio das consultas de pré-natal, com a finalidade de prevenir a pré-eclâmpsia, eclâmpsia e a fase crítica da síndrome HELLP. A hipertensão na gestante é silenciosa e por isso deve ser acompanhada e tratada precocemente. É fundamental que seja identificada ainda no pré-natal de pacientes com gestação de alto risco, pois precisam de assistência específica e direcionada para a prevenção de complicações.

Uma das complicações da pré-eclâmpsia é a síndrome HELLP, que pode ocorrer durante a gestação, no trabalho de parto ou no puerpério imediato. A associação de hemólise, plaquetopenia e disfunção hepática já era relatada na década de 50. Em 1982, Weinstein reuniu essas alterações sob o acrômio de síndrome HELLP. Identificou esta síndrome como uma complicação da pré-eclâmpsia, cujas características são hipertensão, edema e proteinúria. O diagnóstico precoce é, eminentemente laboratorial, e deve ser pesquisado de maneira sistemática nas mulheres com pré-eclâmpsia e sintoma de dor em hipocôndrio direito (Nery et al., 2014).

A síndrome de HELLP pode evoluir para complicações graves nas gestantes, sua fisiopatologia ainda não é totalmente esclarecida e o diagnóstico difícil de ser realizado. A mortalidade é elevada e o tratamento, além da cesárea, ainda não é específico (Pereira et al., 2019). Os sinais e sintomas de HELLP são: edema, aumento na pressão arterial, proteinúria, cefaleia, algia, náuseas e vômitos. Tais sintomas devem ser observados com atenção e controlados para prevenir a forma mais grave da doença, como a SHEG, que pode levar a alterações no desenvolvimento do feto, morte fetal e/ou morte da gestante (Mendes et al., 2021).

O enfermeiro precisar estar atento as complicações associadas a síndrome de $H E L L P$, pois o agravo da síndrome hipertensiva da gestação, pré-eclâmpsia e eclâmpsia, apresenta relevância clínica e aspectos como alterações exames laboratoriais, sinais e sintomas apresentados e relatados. Durante o cuidado a gestante, o enfermeiro deve promover conforto físico, bem-estar, controle das queixas álgicas, sinais e sintomas apresentados (Nour et al., 2015). 
A síndrome HELLP se desenvolve rapidamente e é decorrente de uma pré-eclâmpsia já existente, o que dificulta muito o trabalho do profissional de saúde ao realizar cuidados específicos com a paciente. Ao considerar esta problemática, surgiu o seguinte questionamento de pesquisa: quais são os cuidados de enfermagem em gestante com síndrome de HELLP? O objetivo central deste estudo é descrever os cuidados de enfermagem na gestante com síndrome de HELLP e, mais especificamente, descrever as principais complicações da síndrome de HELLP na gestante.

\section{Metodologia}

O estudo foi realizado por meio de uma revisão bibliográfica de abordagem descritiva e qualitativa, que é descrita por Cesário, Flauzino e Mejia (2020) como um tipo de pesquisa baseada em material já construído, como monografias ou artigos publicados em periódicos científicos. Inicialmente buscou-se reunir evidências para responder à pergunta de pesquisa e nessa primeira etapa a busca foi realizada em bibliotecas digitais, que permitem uma busca em bases de dados próprias por meio da utilização de descritores padronizados.

Encontrou-se no DeCS (Descritores em Ciências da Saúde) os seguintes descritores: cuidados de enfermagem, síndrome hellp e papel do profissional de enfermagem. Os bancos de dados utilizados foram: No Google acadêmico, utilizouse os descritores entre aspas ("') e o operador lógico booleano "AND” e "OR". Na BVS (biblioteca virtual em saúde), foi utilizado a opção pesquisa avançada e foram selecionadas as bases da BDENF (Banco de Dados em Enfermagem), LILACS (Literatura Latino-Americana e do Caribe em Ciências da Saúde) e o operador lógico booleano "OR" e "AND". Na SciELO (Scientific Electronic Library Online), foi utilizada a opção pesquisa avançada e o operador lógico booleano "OR" e "AND".

Estabeleceu-se como critérios de inclusão, artigos acadêmicos publicados entre 2013 e 2021, na língua portuguesa, disponíveis de forma gratuita e nos bancos de dados já mencionados, que respondessem à pergunta de pesquisa. Excluíram-se artigos repetidos encontrados nas bases de dados, resumos, artigos inferiores a 2013 e artigos que não respondiam o problema da pesquisa. A coleta dos dados foi realizada no mês de abril por três pesquisadores de forma independente. Os resultados das buscas pelos dados e do número final de publicações que fizeram parte da revisão são apresentados conforme o fluxograma de Prisma (Peters, 2015), como mostra a Figura 1: 
Figura 1. Diagrama de fluxo dos artigos encontrados.

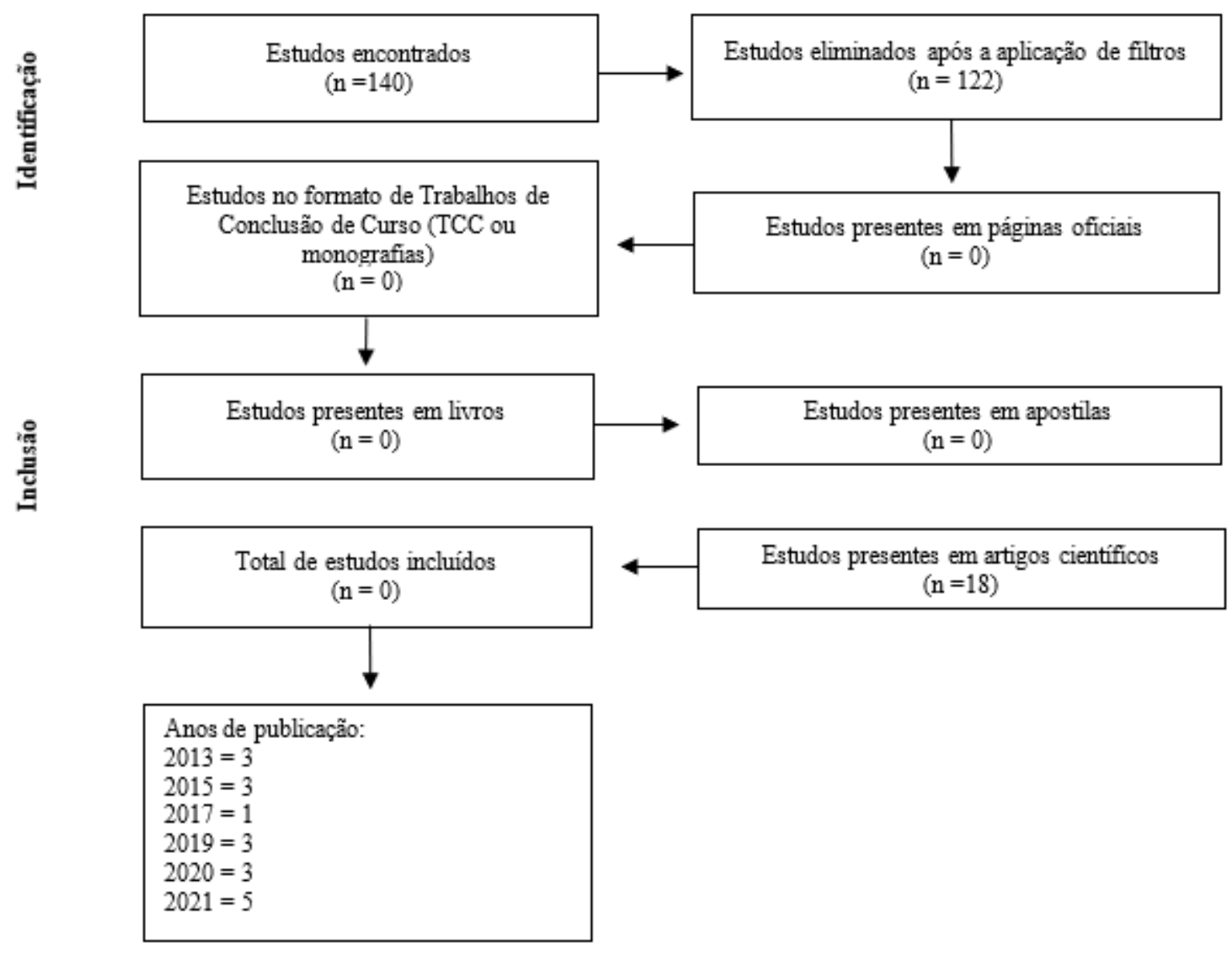

Fonte: elaborado pelos autores (2021).

\section{Resultados}

Para uma melhor organização dos artigos foi desenvolvida a Tabela 1, que mostra a distribuição inicial dos artigos científicos encontrados nas bases de dados da BVS, ScIELO e Google Acadêmico. É possível notar que o maior aproveitamento de periódicos foi na base do Google Acadêmico com 10 artigos incluídos, na BVS, 5 periódicos foram inclusos e na ScIELO, 3 artigos foram aproveitados, o que resultou em uma amostra final de 18 artigos científicos.

Tabela 1. Artigos inclusos neste estudo, por base de dados

\begin{tabular}{|lclclcr|} 
BVS & & ScIELO & & \multicolumn{2}{l}{ Google Acadêmico } & Amostra final \\
\hline Total & 50 & Total & 30 & Total & 60 & \\
Excluídos & 32 & Excluídos & 27 & Excluídos & 50 & 18 artigos \\
\hline Incluídos & 5 & Incluídos & 3 & Incluídos & 10 & \\
\hline
\end{tabular}

Fonte: Autores (2021).

O Quadro 1 foi desenvolvido para apresentar um melhor entendimento dos periódicos selecionados nas bases de dados, pois permite a comparação entre os assuntos abordados em cada um dos estudos selecionados. Para a elaboração do quadro foram utilizadas as seguintes variáveis: autor, título, objetivo central e tipo de estudo. No Quadro 1 é possível notar a relação dos artigos científicos com os objetivos da pesquisa: 
Quadro 1. Artigos incluídos neste estudo.

\begin{tabular}{|c|c|c|c|}
\hline Autor & Título & Objetivo & Método \\
\hline Oliveira et al., 2017 & $\begin{array}{l}\text { Assistência de enfermeiros na } \\
\text { síndrome hipertensiva } \\
\text { gestacional em hospital de } \\
\text { baixo risco obstétrico. }\end{array}$ & $\begin{array}{l}\text { Analisar a assistência de enfermeiros às } \\
\text { gestantes com síndrome hipertensiva, } \\
\text { em um hospital de baixo risco } \\
\text { obstétrico. }\end{array}$ & $\begin{array}{l}\text { Pesquisa de campo, } \\
\text { descritiva, exploratória, com } \\
\text { abordagem qualitativa. }\end{array}$ \\
\hline Pereira et al., 2019 & $\begin{array}{l}\text { Síndrome HELLP: uma revisão } \\
\text { de literatura. }\end{array}$ & $\begin{array}{l}\text { Compreender melhor sobre a } \\
\text { fisiopatologia, diagnóstico e tratamento } \\
\text { da Síndrome HELLP. }\end{array}$ & Revisão de literatura. \\
\hline $\begin{array}{l}\text { Krebs, Silva, Bellotto, } \\
2021\end{array}$ & $\begin{array}{l}\text { Síndrome de HELLP } \mathrm{e} \\
\text { Mortalidade Materna: Uma } \\
\text { revisão integrativa. }\end{array}$ & $\begin{array}{l}\text { Pesquisar a mortalidade materna em } \\
\text { pacientes com Síndrome HELLP. }\end{array}$ & Pesquisa integrativa. \\
\hline Abrahão. et al., 2020 & $\begin{array}{l}\text { Atuação do enfermeiro a } \\
\text { pacientes } \\
\text { síndrome } \\
\text { específica da gestação atuação } \\
\text { do enfermeiro a pacientes } \\
\text { portadoras de } \\
\text { hipertensiva } \\
\begin{array}{l}\text { hipertensiva } \\
\text { gestação. }\end{array}\end{array}$ & $\begin{array}{l}\text { Identificar a importância da assistência } \\
\text { de enfermagem às gestantes com } \\
\text { Síndrome Hipertensiva Gestacional. }\end{array}$ & $\begin{array}{l}\text { Estudo bibliográfico, } \\
\text { descritivo e exploratório. }\end{array}$ \\
\hline Campos. et al., 2019 & $\begin{array}{l}\text { Conhecimento de enfermeiros } \\
\text { sobre a doença hipertensiva } \\
\text { especifica da gestação. }\end{array}$ & $\begin{array}{l}\text { Possível identificar as condutas dos } \\
\text { enfermeiros com pacientes de DHEG. }\end{array}$ & Revisão integrativa. \\
\hline $\begin{array}{l}\text { Antônio, } \\
\text { Galdino. } 2019\end{array}$ & $\begin{array}{l}\text { O conhecimento das gestantes } \\
\text { sobre síndrome hipertensiva } \\
\text { específica da gravidez (SHEG), }\end{array}$ & $\begin{array}{l}\text { Analisar o conhecimento das gestantes } \\
\text { sobre Síndrome Hipertensiva Específica } \\
\text { da Gravidez. }\end{array}$ & $\begin{array}{l}\text { Estudo } \\
\text { exploratório de abordagem } \\
\text { qualitativa. }\end{array}$ \\
\hline Mendes. et al., 2021 & $\begin{array}{l}\text { Vivência acadêmica da } \\
\text { assistência de enfermagem á } \\
\text { mulheres com síndrome } \\
\text { hipertensiva durante a gravidez } \\
\text { atendidas em uma maternidade } \\
\text { em Belém do Pará: um relato de } \\
\text { experiência. }\end{array}$ & $\begin{array}{l}\text { Descrever a vivência acadêmica de } \\
\text { alunos do curso de enfermagem, na } \\
\text { assistência às mulheres com síndrome } \\
\text { hipertensiva durante a gestação em } \\
\text { tratamento em uma maternidade de } \\
\text { referência de Belém do Pará. }\end{array}$ & Relato de experiência. \\
\hline Couto. et al., 2020 & $\begin{array}{l}\text { Conhecimento de enfermeiros } \\
\text { da atenção básica na detecção } \\
\text { precoce da síndrome HELLP. }\end{array}$ & $\begin{array}{llll}\text { Analisar o conhecimento } & \text { de } \\
\text { enfermeiros da atenção básica } & \text { na } \\
\text { detecção da Síndrome HELLP. } & \end{array}$ & Estudo qualitativo. \\
\hline Lopes. et al., 2013 & $\begin{array}{l}\text { Hipertensão gestacional e a } \\
\text { síndromeHELLP: ênfase nos } \\
\text { cuidados de enfermagem. }\end{array}$ & $\begin{array}{l}\text { Fatores de risco para a síndrome } \\
\text { HELLP. }\end{array}$ & Revisão integrativa. \\
\hline Sampaio. et al., 2013 & $\begin{array}{l}\text { Cuidados de enfermagem } \\
\text { prestados a mulheres com } \\
\text { hipertensão gestacional e pré- } \\
\text { eclâmpsia. }\end{array}$ & $\begin{array}{l}\text { Descrever e analisar a importância dos } \\
\text { cuidados de enfermagem que devem ser } \\
\text { prestados a mulheres com hipertensão } \\
\text { gestacional/pré-eclâmpsia tendo em } \\
\text { vista seu diagnóstico precoce e a } \\
\text { identificação } \text { de } \text { possíveis }\end{array}$ & Revisão da literatura. \\
\hline
\end{tabular}


Research, Society and Development, v. 10, n. 8, e47810817669, 2021

(CC BY 4.0) | ISSN 2525-3409 | DOI: http://dx.doi.org/10.33448/rsd-v10i8.17669

\begin{tabular}{|c|c|c|c|}
\hline & & complicações. & \\
\hline Nour et al., 2015 & $\begin{array}{l}\text { Mulheres com síndrome } \\
\text { hipertensiva específica da } \\
\text { gravidez: evidências para o } \\
\text { cuidado de enfermagem. }\end{array}$ & $\begin{array}{l}\text { Identificar as evidências disponíveis na } \\
\text { literatura sobre os conhecimentos e } \\
\text { sentimentos de mulheres com síndrome } \\
\text { hipertensiva específica da gravidez } \\
\text { (SHEG) e os cuidados de enfermagem. }\end{array}$ & Revisão integrativa. \\
\hline Marciano et al., 2020 & $\begin{array}{l}\text { O papel da equipe de } \\
\text { enfermagem frente a crise } \\
\text { hipertensiva. }\end{array}$ & $\begin{array}{l}\text { Descrever a assistência de enfermagem } \\
\text { frente a uma crise hipertensiva e } \\
\text { classificá-la de acordo com suas } \\
\text { características fisiopatológicas. }\end{array}$ & Revisão literária \\
\hline Freires. et al., 2013 & $\begin{array}{l}\text { Percepção da gestante pré- } \\
\text { eclâmptica quanto a assistência } \\
\text { de enfermagem durante o } \\
\text { período de hospitalização. }\end{array}$ & $\begin{array}{l}\text { Analisar a assistência de enfermagem } \\
\text { prestada à gestante com pré-eclâmpsia. }\end{array}$ & Estudo exploratório. \\
\hline Brito et al., 2015 & $\begin{array}{l}\text { Prevalência das síndromes } \\
\text { hipertensivas específicas da } \\
\text { gestação (SHEG), }\end{array}$ & $\begin{array}{l}\text { Identificar a prevalência das Síndromes } \\
\text { Hipertensivas Específica da Gestação e } \\
\text { traçar o perfil epidemiológico das } \\
\text { gestantes. }\end{array}$ & Pesquisa retrospectiva. \\
\hline $\begin{array}{l}\text { Nascimento, Bocardi, } \\
\text { Rosa, } 2015\end{array}$ & $\begin{array}{l}\text { Doença hipertensiva específica } \\
\text { da gravidez (DHEG) em } \\
\text { adolescentes: uma revisão de } \\
\text { literatura. }\end{array}$ & $\begin{array}{l}\text { Analisar as publicações relacionadas à } \\
\text { DHEG na adolescência. }\end{array}$ & Estudo descritivo. \\
\hline Silva et al., 2021 & $\begin{array}{l}\text { Assistência de enfermagem às } \\
\text { mulheres com pré-eclâmpsia: } \\
\text { revisão integrativa }\end{array}$ & $\begin{array}{l}\text { Levantar evidências científicas sobre a } \\
\text { assistência de enfermagem à mulher } \\
\text { com pré-eclâmpsia. }\end{array}$ & Estudo descritivo. \\
\hline Fialho et al., 2021 & $\begin{array}{l}\text { Identificação do perfil } \\
\text { epidemiológico e dos fatores de } \\
\text { risco prégestacionais } \\
\text { gestacionais relacionados ao } \\
\text { desenvolvimento da síndrome } \\
\text { HELLP }\end{array}$ & $\begin{array}{l}\text { Descrever o perfil epidemiológico e } \\
\text { identificar os fatores de risco pré- } \\
\text { gestacionais e gestacionais relacionados } \\
\text { ao desenvolvimento da síndrome } \\
\text { HELLP. }\end{array}$ & $\begin{array}{l}\text { Estudo documental, } \\
\text { descritivo, retrospectivo e } \\
\text { quantitativa. }\end{array}$ \\
\hline Sousa et al., 2021 & $\begin{array}{l}\text { Atuação da enfermagem no } \\
\text { atendimento às emergências } \\
\text { obstétricas: Eclâmpsia e Pré- } \\
\text { eclâmpsia }\end{array}$ & $\begin{array}{l}\text { Analisar a atuação da equipe de } \\
\text { enfermagem no atendimento às } \\
\text { emergências obstétricas: eclampsia e } \\
\text { pré-eclâmpsia. }\end{array}$ & $\begin{array}{l}\text { Revisão bibliográfica } \\
\text { integrativa. }\end{array}$ \\
\hline
\end{tabular}

Fonte: Autores (2021).

\section{Discussão}

\section{Alterações fisiológicas ocasionada pela $H E L L P$}

No período gestacional ocorrem transformações anatômicas e fisiológicas no corpo da mulher, este processo se inicia nas primeiras semanas de gestação e termina após o parto. Durante o período de formação do feto a gestante requer um acompanhamento médico e de enfermagem para prevenir possíveis complicações como a hipertensão gestacional, que pode ocasionar várias disfunções fisiopatológicas como: alteração das funções hepáticas, hematológicas, hidroeletrolíticas, uteroplacentárias e danos cerebrais (Oliveira. et al. 2017). 
Conforme Brito et al. (2015), a SHEG ocorre próximo à $20^{\text {a }}$ semana gestação e pode apresentar 3 fases distintas, denominadas de pré-eclâmpsia, eclampsia e síndrome de HELLP. Na pré-eclâmpsia ocorre a hipoperfusão e mal formação placentária, disfunção endotelial, alteração das enzimas de coagulação e diminuição do fluxo sanguíneo na placenta. Os sintomas da pré-eclâmpsia são: dor abdominal no quadrante superior direito, vômitos náuseas, elevação da pressão arterial, edema e aumento das proteínas na urina (Pereira et al., 2019).

$\mathrm{Na}$ eclâmpsia ocorre a elevação da pressão arterial, diminuição do fluxo sanguíneo, vasoconstrição placentária, mal formação placentária em casos graves o feto evolui para óbito intrauterino. Os sinais e sintomas que a paciente pode apresentar são: distúrbios gástricos como vomito e náuseas, alterações no sistema nervoso central, convulsões, cefaleia, perca da consciência, encefalopatias hipertensivas, vaso espasmo cerebral, AVC (acidente vascular encefálico), lesão endotelial e edema vasogênico (Abrahão et al., 2020 \& Sousa et al., 2021).

Krebs, Silva e Bellotto (2021) afirmam que a síndrome de HELLP é o agravamento da pré-eclâmpsia ou eclampsia e apresenta as seguintes características: hemólise de células sanguíneas, alterações das funções hepáticas e diminuição do número de plaquetas que circulam no sangue. Conforme Lopes et al. (2013), os principais fatores de risco para a gestante desenvolver a síndrome de HELLP está associada casos crônicos de hipertensão, idade, etnia branca, histórico de problemas obstétricos, gemelaridade, multiparidade, pré-eclâmpsia, eclampsia e diabetes mellitus. Nour et al. (2015), afirma que para realizar o diagnóstico da síndrome de HELLP são necessários exames laboratoriais que comprovem alterações na função hepática, coagulação e hemólise, pois os sinais e sintomas podem ser confundidos com a pré-eclâmpsia e eclâmpsia. O principal tratamento para a síndrome de HELLP é realizar o parto cesariano mais breve possível.

A gestante com síndrome de HELLP também pode desenvolver outras doenças como a esteatose hepática, pancreatite aguda, púrpura trombocitopenia idiopática, lúpus sistêmicos, síndrome antifosfolipídico e colelitíase (Pereira et al., 2019). Outras complicações relacionadas a síndrome incluem: problemas cerebrovasculares, hemorragia, edema pulmonar, descolamento da retina, hematoma/hepática, insuficiência renal aguda, insuficiência hepática, insuficiência coagulopatia, desprendimento placentário, sepse, ativação plaquetária, elevação níveis séricos ocitocinas, obstrução sinusoidal e infarto hepático, hemorragia hepática, contrações uterinas, convulsões e hemólise (Couto et al., 2020).

\section{Prevenção da HELLP}

Os sinais e sintomas da SHEG devem ser identificados no pré-natal, conforme as seguintes alterações: hipertensão arterial, edema de membros e facial, proteinúria, desconforto para deambular e respiratório, cefaleia acompanhada de anormalidade visuais, epigastralgia, plaquetopenia, elevação da enzima hepática. Com esses sinais e sintomas a gestante deve ser encaminhada para pré-natal de alto risco, pode poderá apresentar tendência maior para desenvolver a doença durante o andamento de sua gestação. Um acompanhamento especializado poderá conter complicações no quadro. O enfermeiro deve orientar sobre mudanças no estilo de vida, esclarecer as dúvidas sobre pré-natal de alto risco, monitorar sinais e sintomas apresentados, estimular a paciente a aderir a um plano alimentar saudável e identificar o agravamento da doença (Campos $e t$ al., 2019).

O enfermeiro é responsável por traçar todo o plano assistencial de enfermagem, além de gerenciar e coordenar a prática clínica de sua equipe, é um dos profissionais que desenvolve as condutas preventivas para a SHEG com o controle e educação em saúde, principalmente na atenção primária para a gestante. As consultas do pré-natal, realizadas por enfermeiros, são essenciais para acompanhar e orientar as gestantes e puérperas sobre o controle de doenças hipertensivas (Mendes et al., 2021)

Silva et al. (2021) descreve que o enfermeiro ao realizar a consulta de pré-natal precisa ficar atento as manifestações clínicas e as complicações apresentadas pelo quadro hipertensivo, além de ofertar o apoio emocional para melhorar o hábito 
alimentar e estimular a prática de exercício diário para diminuir as possíveis complicações e agravos relacionados da SHEG. (Couto et al., 2020)

\section{Assistência de enfermagem na HELLP}

Por meio da Sistematização da Assistência de Enfermagem (SAE), o enfermeiro pode se basear em um método científico para auxiliar na identificação das situações de risco para a saúde para a gestante (Nascimento, Bocardi \& Rosa, 2015). Aguiar et al. (2010) descreve que a SAE pode ser operacionalizada pelo processo de enfermagem, um sistema composto de cinco etapas: histórico, diagnósticos, planejamento, implementação (intervenções de enfermagem) e avaliação. A assistência de enfermagem deve ser individualizada à gestante com HELLP, dessa forma é necessário que o enfermeiro realize os diagnósticos de enfermagem que melhor atenta as necessidades da paciente, além de implementar um plano de intervenção eficaz, que possa ser avaliado constantemente e ajustado sempre que necessário (Brito et al., 2015).

Os principais diagnósticos de enfermagem encontrados na literatura que se encaixam no perfil da gestante com HELLP são apresentados no Quadro 2, que dois possui duas variáveis: diagnósticos de enfermagem e fatores relacionados.

Quadro 2. Diagnóstico de enfermagem em gestantes com HELLP e fatores relacionados.

\begin{tabular}{|l|l|}
\hline \multicolumn{1}{|c|}{ Diagnósticos de enfermagem } & \multicolumn{1}{c|}{ Fatores relacionados } \\
\hline Risco de infecção & Procedimento invasivos, acesso periféricos, sondagem vesical de demora \\
\hline Dor aguda & Cefaleia, epigastralgia, dor hipocôndrio e baixo ventre. \\
\hline Baixa autoestima & $\begin{array}{l}\text { ausência da família e parceiro (casos de internações prolongada), incerteza do que irá } \\
\text { acontecer, aparência. }\end{array}$ \\
\hline Volume de líquido excesso & Edema ocasionada pela patologia, controle e monitorização hídrico e eletrólitos \\
\hline Náusea & $\begin{array}{l}\text { Devido ao aumento da progesterona que ocasiona a diminuição motilidade tônus } \\
\text { gástricos, refluxos gastresofágicos. }\end{array}$ \\
\hline Privação do sono & Ansiedade, estresse ocasionado pela patologia, desconforto físico. \\
\hline Risco de disfunção hepática & Elevação dos níveis TGO e TGP (pode ocasionar dano hepático, edema e hemorragia). \\
\hline Eliminação urinaria prejudicada & Diminuição na taxa de filtração glomerular e perfusão renal. \\
\hline Constipação & Diminuição motilidade tônus gástricos. \\
\hline Nutrição desiquilibrada & $\begin{array}{l}\text { Alteraçães dos hábitos alimentares por diversos motivos, como a náusea, pirose, } \\
\text { mudanças do padrão alimentar. }\end{array}$ \\
\hline Ansiedade & Medo da morte e alteração da rotina diária \\
\hline Risco de infecção & Procedimento invasivos, acesso periféricos, sondagem vesical de demora \\
\hline
\end{tabular}

Fonte: Autores (2021).

Após a elaboração do quadro acima, foi possível identificar as seguintes intervenções de enfermagem na paciente com síndrome de HELLP: avaliação rigorosa dos sinais vitais de 2 em 2 horas, avaliação constante do débito urinário, verificação dos reflexos, controle dos batimentos cardiofetal, manter o paciente em decúbito lateral esquerdo, atentar-se para cefaleia, distúrbio visual, dor epigástrica e verificar constantemente o nível de consciência. A assistência de enfermagem à gestante com HELLP também consiste em manter o repouso, controle dos sinais vitais, pesar diariamente, controle hidroeletrolítico e controle dos batimentos fetais e realizar controles dos exames laboratoriais (Nascimento, Bocardi \& Rosa, 2015).

O enfermeiro, junto com a equipe de enfermagem, desempenha uma função fundamental, pois atende de forma humanizada, com suporte psicológico, fornece informações para ouvir, esclarecer dúvidas, angústias, medos, anseios e esperanças da gestante, com objetivo de garantir um melhor prognóstico. A equipe de enfermagem deve atentar-se a elevação 
da pressão arterial igual ou superior 140/90 mmHg, ganho de peso, edema, algia abdominal, retenção urinária, vertigem e proteínas encontrada na urina por meio exame laboratorial (Freires et al., 2013).

\section{Conclusão}

A síndrome de HELLP é caracterizada pela hemólise, elevação das enzimas hepáticas e trombocitopenia e pode apresentar complicações cerebrovasculares, hemorragia, edema pulmonar, descolamento da retina, hematoma/hepática, insuficiência renal aguda, insuficiência hepática, insuficiência coagulopatia, desprendimento placentário, sepse, ativação plaquetária, elevação níveis séricos ocitocinas, obstrução sinusoidal e infarto hepático, hemorragia hepática, contrações uterinas, convulsões e hemólise. Devido a gravidade da doença, é necessário um acompanhamento especializado, tanto da equipe médica, quanto pela enfermagem.

Os principais cuidados de enfermagem na gestante com HELLP identificados na literatura analisada foram: monitorizar a frequência respiratória, saturação de oxigênio, débito urinário e reflexos patelares, manter o repouso, pesar diariamente, realizar controle hidroeletrolítico e controlar os batimentos fetais. Durante a elaboração da pesquisa notou-se um uma escassez de publicações nacionais a respeito do assunto abordado e dessa forma, os autores esperam que este estudo contribua para novas pesquisas relacionadas a assistência de enfermagem na paciente com síndrome de HELLP.

\section{Referências}

Abrahão, A. C. M., Santos, R. F. S., Viana, S. R. G., Viana, S. M., \& Costa, C. S. C. (2020), Atuação do enfermeiro a pacientes portadoras de síndrome hipertensiva específica da gestação atuação do enfermeiro a pacientes portadoras de síndrome hipertensiva específica da gestação. Revista Cientifica Escola Estadual Saúde Pública Goiás “Candido Santiago”. 6(1), 51-63.

Antônio, E. D. A. P., Pereira, T. V., \& Galdino, C. V. (2019), O conhecimento das gestantes sobre síndrome hipertensiva específica da gravidez (SHEG), Saber Digital Revista eletrônica CESVA. 12(1), 1-13.

Brito, K. K. G., Moura, J. R. P., Merifane, J., Brito, J. V. O., Santos, S. H., \& Soares, M. J. G. O. (2015), Prevalência das síndromes hipertensivas específicas da gestação (SHEG), Revista de Pesquisa Cuidado é Fundamental Online. 7(3), 2717-2725.

Campos, L., Gomes, E. A., Silva, D. C. F., \& Berlet, L. J. (2019), Conhecimento de enfermeiros sobre a doença hipertensiva especifica da gestação. Revista Saúde Viva Multidisciplinar da AJES. 2(2), 25-38.

Cesário, J. M. S., Flauzino, V. H. P., \& Mejia, J. V. C. (2020) Metodologia científica: Principais tipos de pesquisas e suas caraterísticas. Revista Científica Multidisciplinar Núcleo do Conhecimento. 5(11). 23-33. http://dx.doi.org/10.32749/nucleodoconhecimento.com.br/educacao/tipos-de-pesquisas.

Couto, P. L. S. C., Piava, M. S., Chaves, V. M., Benemérita, A., Vilela, A., Santos, N. R., Pereira, S. S. C., Antunes, I. F. R. S., Ferreira, L. C., \& Merces, M. C. (2020), Conhecimento de enfermeiros da atenção básica na detecção precoce da síndrome HELLP. Revista Saúde Santa Maria. 46(1), 1-15. http://dx.doi.org/10.5902/2236583439353.

Freires, M. B., Alencar, J. S., Alencar, L. S., \& Saraiva, E. M. S. (2013), Percepção da gestante pré-eclâmptica quanto a assistência de enfermagem durante o período de hospitalização. Id on line Revista de Psicologia. 7(19), 44-53. http://dx.doi.org/10.14295/idonline.v7i19.223.

Krebs, V. A., Silva, M. R., \& Bellotto, P. C. B. (2021), Síndrome de Hellp e Mortalidade Materna: Uma revisão integrativa. Brazilian Journal of Health Review. 4(2), 6297-6311. http://dx.doi.org/10.34119/bjhrv4n2-184.

Lopes, G. T., Oliveira, M. C. R., Silva, K. M., Silva, I. F., \& Ribeiro, A. P. L. P. (2013), Hipertensão gestacional e a síndrome HELLP: ênfase nos cuidados de enfermagem. Revista Augustus. 18(36), 77-89.

Marciano, M. V. F., Assis, L. M., Beserra, F. F., \& Bacelar, L. F. F. (2020), O papel da equipe de enfermagem frente a crise hipertensiva. Brazilian Journal of Surgery and Clinical Research. 33(3), 87-93.

Mendes, A. P. S., Bezerra, A. L. L., Martins, C. F., Barros, B. T. D., Palheta, D. R. A., Soares, L. S., Castro, T. R. A., \& Vilhena, V. F. S. (2021), Vivência acadêmica da assistência de enfermagem á mulheres com síndrome hipertensiva durante a gravidez atendidas em uma maternidade em Belém do Pará: um relato de experiência. Brazilian Journal of Health Review. 4(2), 4704-4709. http://dx.doi.org/10.34119/bjhrv4n2-057.

Nascimento, T. L. C., Bocardi, M. I. B., \& Rosa, M. P. R. S. (2015), Doença hipertensiva específica da gravidez (DHEG) em adolescentes: uma revisão de literatura. Ideias \& Inovação. 2(2), 69-76.

Nery, I. S., Viana, L. S., Viana, L. M. M., Araujo, T. M. E., Feitosa, V. C., \& Pereira, V. F. (2014), Perfil epidemiológico e obstétrico de gestantes com síndrome HELLP. Cogitare Enfermagem. 19(1), 147-152.

Nour, G. F. A., Castro, M. M., Fontenele, F. M. C., Olievira, M. S., Brito, J. O., \& Oliveira, A. R. S. (2015), Mulheres com síndrome hipertensiva específica da gravidez: evidências para o cuidado de enfermagem. Sanare. 14(1),121-128. 
Research, Society and Development, v. 10, n. 8, e47810817669, 2021

(CC BY 4.0) | ISSN 2525-3409 | DOI: http://dx.doi.org/10.33448/rsd-v10i8.17669

Oliveira, G. S., Paixão, G. P. N., Franga, C. D. S., Santos, M. K. R., \& Santos, M. A. (2017), Assistência de enfermeiros na síndrome hipertensiva gestacional em hospital de baixo risco obstétrico. Revista Cuidarte. 8(2), 1561-72. http://dx.doi.org/10.15649/cuidarte.v8i2.374.

Pereira, B., Linhares, G. M. M., Severo, I. M. M., Serra, J. L. G., Dutra, M., Quintão, R. A., \& Carvalho, J. (2019), Síndrome HELLP: uma revisão de literatura. Revista da Faculdade de Medicina de Teresópolis. 3(2), 61- 68.

Pollo-Flores, P., Ferraz, L., Lopes, P. F., Marcolino, L., Amin-Junior, J., Rezende-Filho, J., \& Braga, A. (2015), Alterações hepáticas da gravidez. Revista Femina. 43(5), 225-234

Ribeiro, J. F., Soares, M. S. A. C., Rodrigues, C. O., Bezerra, V. O. R., \& Araujo, K. R. S. (2016), Perfil sociodemográfico e clínico de mulheres com síndrome HELLP. Rev. Enferm. UFSM. 6(4), 569-577. http://dx.doi.org/10.5902/2179769221345.

Sampaio, T. A. F., Santana, T. D., Hanzelmann, R. S., Santos, L. F. M., Montenegro, H. R. A., Martins, J. S. A., Helena, A. A. S., \& Ferreira, D. C. (2013), Cuidados de enfermagem prestados a mulheres com hipertensão gestacional e pré-eclâmpsia. Revista Saúde Física \& Mental. 2(1), $36-45$.

Sousa, R. S. S., Silva, L. A., Santos, E. A., Ferreira, N. K. F., Lima, E. D., Silva, S. K. T., Silva, C. C., \& Silva, A. E. (2021). Atuação da enfermagem no atendimento às emergências obstétricas: Eclâmpsia e Pré-eclâmpsia. Brazilian Journal of Health Review. 4(1), 1022-1032. http://dx.doi.org/10.34119/bjhrv4n1-089. 